\title{
Conmemorar la Ashura en la diáspora. Limitaciones y posibilidades en el uso del espacio público en Barcelona y París
} Commemorate the Ashura in the diaspora. Limitations and possibilities in the use of public space in Barcelona and Paris

Víctor ALBERT BLANCO

Universidad París 8

valbert.blanco@gmail.com

https://orcid.org/0000-0003-4104-8644

Rosa MARTÍNEZ CUADROS

Universidad Autónoma de Barcelona

rosa.martinez.cuadros@uab.cat

https://orcid.org/0000-0001-7768-8374

Recibido 24/07/2020. Aceptado 10/03/2021

Para citar este artículo: Víctor ALBERT BLANCO y Rosa MARTÍNEZ CUADROS (2021): “Conmemorar la Ashura en la diáspora. Limitaciones y posibilidades en el uso del espacio público en Barcelona y París" en Revista de Estudios Internacionales Mediterráneos, 30, pp. 1-23.

Para acceder a este artículo: https://doi.org/10.15366/reim2021.30.001

\section{Resumen:}

La Ashura constituye una de las conmemoraciones más importantes del calendario musulmán chií. Siendo un día en el que se recuerda el sufrimiento del imán Hussein asesinado en 680 DC, la conmemoración incluye una procesión en la vía pública. Desde hace algunos años, no sólo tiene lugar en países como Irán o Pakistán, sino también en grandes ciudades europeas. Este artículo compara la conmemoración de la Ashura en Barcelona y París con el objetivo de analizar las diferentes lógicas que determinan su dimensión transnacional, así como el uso que las comunidades de la diáspora chií pueden hacer del espacio público.

Palabras clave: ashura/ espacio público/ religión/ islam/ diáspora/

Abstract:

Ashura is one of the most important commemorations of the Shiite Muslim calendar. Being a day in which the suffering of Imam Hussein assassinated in $680 \mathrm{AC}$ is remembered, the commemoration 
includes a procession in public space. For some years now, it has not only taken place in countries like Iran or Pakistan, but also in large European cities. This article compares the commemoration of the Ashura in Barcelona and Paris in order to analyze the different logics that determine its transnational dimension, as well as the use that the Shiite diaspora's communities can make of public space.

Keywords: Ashura/ Public Space/ Religion/Islam/ Diaspora/

1 de octubre de 2017. Es el día de la Ashura, una conmemoración chií que, al menos desde el año 2005, la comunidad de Al-Qaim de Barcelona celebra con una procesión en el espacio público. A lo largo de los años, ésta ha sufrido cambios en su itinerario y en su formato, a pesar de haber estado siempre autorizada por parte de las administraciones. Sin embargo, en esta ocasión la procesión no se ha llevado a cabo. Hay un referéndum de autodeterminación convocado por parte del movimiento independentista catalán, y se prevé una jornada de enorme tensión entre votantes y policía. Ante esta situación, unas horas antes del inicio previsto, nos avisan de que se ha cancelado la procesión y que la conmemoración tendrá lugar en un polideportivo cerrado.

Diario de campo, B_01/10/2017

Hacia las 14h algunas decenas de jóvenes de las comunidades chiíes de la región parisina se congregan en la céntrica Plaza de la República de la capital francesa. Como nos explican algunos de ellos, es la primera vez que realizan una actividad así coincidiendo con el día de la Ashura. El evento se presenta como una propuesta cultural sin cabida para rituales religiosos. Éstos se han llevado a cabo en el interior de las distintas mezquitas chiitas de la región a lo largo de la mañana. A diferencia del caso barcelonés, la procesión y los rituales propios de la Ashura siempre se han relegado al espacio privado. Para esta ocasión, los jóvenes chiitas han usado el espacio público simplemente para dar a conocer la conmemoración a través del reparto de folletos, flores y chocolatinas entre los transeúntes.

Diario de campo, P_01/10/2017

Siendo una de las conmemoraciones más importantes del calendario musulmán chií, rama minoritaria del islam, la Ashura es un día en el que se recuerda el sufrimiento y el asesinato del imam Hussein, nieto de Muhammad en el año 680 DC. Esta conmemoración suele incluir una procesión en el espacio público acompañada por distintos rituales. Coincidiendo con el décimo día del Muharram o primer mes del calendario musulmán, la Ashura tiene lugar en los distintos países en los que los chiitas constituyen una comunidad significativa, mayoritaria en algunos casos, como Irán, Irak, Siria, Líbano o Pakistán, siendo un elemento importante de "afirmación" grupal (VivierMuresan, 2020). El mismo día, y como resultado de los flujos migratorios globales y la emergente presencia de comunidades chíes en contextos diaspóricos, la Ashura ha empezado a conmemorarse en ciudades europeas y norteamericanas como las citadas, Barcelona y París, adoptando así una relevante dimensión transnacional.

Una de las principales consecuencias de esta dimensión global es que la Ashura adopta diferentes formas en función tanto de dinámicas internas de cada comunidad como de factores externos. Estos últimos son especialmente relevantes, ya que son los que configuran lo que es posible y lo que 
no en el espacio público de cada contexto particular. Así, en las distintas ciudades existen lógicas diferentes de regulación, acompañamiento y control de la expresión religiosa. A partir de una comparación entre los casos de Barcelona y París, donde hemos realizado trabajo de campo entre 2015 y 2019, analizamos estas lógicas en las que intervienen elementos variados como la legislación sobre la libertad de culto, las normativas locales sobre el uso del espacio público, las concepciones sociales de lo que es tolerable y lo que no o el efecto de otros elementos aparentemente inocuos y neutros como el calendario. Una lectura rápida podría conducir a una clasificación binaria entre dos modelos: uno que promocionaría la diversidad religiosa y su visibilidad, propio de Barcelona, y otro que estaría anclado en una laicidad estricta correspondiente a las ciudades francesas. Sin embargo, con este artículo proponemos un análisis que permite relativizar una visión diacrónica entre los dos modelos, enfatizando el conjunto de aspectos que, teniendo incidencia a nivel local, determinan las distintas formas de conmemorar la Ashura. Tal y como muestran las viñetas etnográficas del principio, estas formas son resultado de múltiples procesos y factores complejos.

En este sentido, el análisis de la Ashura amplía la comprensión sobre la relación entre espacio público y religión (Casanova, 1994; Becci et al., 2013) en contextos urbanos supuestamente seculares, mostrando los factores y procesos que contribuyen a definir la forma que adopta dicha conmemoración en cada ciudad. Este artículo expone una práctica religiosa condicionada social y políticamente, que se adapta a unas limitaciones, impuestas o negociadas, a través de un juego de resistencias e innovaciones entre actores religiosos y seculares. Estudiar la Ashura en contextos diaspóricos contribuye pues a entender la religión y las expresiones que se derivan de ésta como objetos socialmente construidos (Beckford, 2003), confirmando además la importancia creciente del rol de las ciudades en su regulación, sin olvidar que éste puede seguir condicionado por lógicas nacionales y globales que trascienden el ámbito estrictamente local (Martínez-Ariño, 2018).

El artículo está organizado en cinco secciones. La primera consiste en una contextualización de la investigación dentro de la literatura existente, y la segunda presenta la metodología utilizada, así como una breve justificación de la elección de los casos. Seguidamente, en el tercer apartado exponemos las descripciones etnográficas de las dos ciudades. En la cuarta sección discutimos los resultados etnográficos y las entrevistas, identificando tres tipos de cuestiones externas: la retórica oficial sobre las expresiones religiosas; las normativas del espacio público; y un calendario marcado por elementos sociales por encima del hecho religioso y urbano. Finalmente, concluimos el artículo con una breve recapitulación de las principales características de la discusión de los resultados.

\section{Religión y espacio público: una gobernanza compleja y multinivel}

La interacción entre religión y espacio urbano está enormemente condicionada por procesos sociales complejos como la secularización y la globalización (Becci et al, 2017). Una parte de las contribuciones académicas sobre esta relación se ha centrado en las estrategias de los propios actores religiosos analizando, por ejemplo, cómo llevan a cabo una apropiación material y simbólica del espacio urbano, lo que algunos autores han identificado como un proceso de "place-making" (Vasques and Knott, 2014; Becci et al, 2017). En las ciudades europeas contemporáneas esta estrategia es visible a través de la proliferación de eventos religiosos en el espacio público, la aparición de tiendas especializadas o la apertura de centros de culto. Para las religiones consideradas minoritarias es una forma de hacerse visibles, reclamar derechos y reafirmar su identidad (Saint-Blancat, 2019), contribuyendo así a un proceso de "desprivatización" a partir de estrategias de contestación y legitimación en el espacio público (Casanova, 1994). 
Una de las religiones que más ha centrado la atención en el ámbito académico ha sido el islam, tanto por su presencia creciente en el contexto europeo como por los debates sociales que ha suscitado, conllevando muchas veces la aplicación de políticas públicas específicas y estrategias de gobernanza particulares por parte de las administraciones. Una parte de literatura ha analizado las dinámicas internas y otras características de las comunidades, discutiendo aspectos como el papel de las segundas generaciones (Duderija, 2007) o la evolución de ciertas prácticas e identificaciones religiosas (Voas and Fleischmann, 2012; Jeldfort, 2011; Roy, 2013).

Otra línea ha centrado la mirada en el rol de las administraciones y los conflictos sociales y políticos que se han derivado de la visibilidad del islam, ya sea a nivel europeo o a escala estatal y local (Göle, 2015; Planet, 2018). Algunos de los aspectos que más se han analizado son la apertura de centros de culto (Astor, 2017; Moreras, 2009; Allievi, 2009; Cesari, 2003 y 2004) o las regulaciones sobre el velo (Bowen, 2007) y el velo integral (Griera y Burchardt, 2016). Con este artículo contribuimos a los debates de esta literatura centrándonos en una expresión religiosa menos conocida protagonizada además por una minoría dentro del islam: la conmemoración de la Ashura por parte de los chiitas.

Partiendo de estas contribuciones, y adoptando una perspectiva constructivista sobre la religión (Beckford, 2003) creemos importante analizar el papel que juegan las administraciones en la definición de las expresiones religiosas y su legitimidad. Tal y como destaca Berg (2019) con el concepto de "secular-place making", es importante identificar los actores y los aspectos autoidentificados como seculares que participan de la construcción de un espacio para lo religioso. En este sentido, consideramos que es clave analizar las regulaciones y las limitaciones impuestas o negociadas por los poderes públicos sobre las expresiones religiosas, determinando cuáles pueden llevarse a cabo en el espacio público, cuáles deben quedar relegadas al espacio privado o cuáles deben ser simplemente prohibidas. En este proceso, tal y como propone Martinez-Ariño (2018), las ciudades juegan un papel cada vez más importante, convirtiéndose en verdaderos espacios políticos en la gestión de la pluralidad religiosa. De este modo, en este artículo analizamos las características particulares de dos casos locales, Barcelona y París, observando como la gobernanza de la diversidad religiosa afecta la conmemoración de la Ashura.

En el caso de Barcelona, la ciudad ha protagonizado una evolución importante en la gestión de la pluralidad religiosa (Griera, 2012), ligada a la emergencia de nuevos grupos y comunidades invisibles hasta hace apenas unas décadas (Estruch et al., 2004). Con la crisis del paradigma de la secularización, a finales de los años 90 del siglo XX, el Ayuntamiento de Barcelona fue pionero en la creación de una Oficina de Asuntos Religiosos (OAR) para poder llevar a cabo una serie de políticas públicas en relación con las minorías religiosas. Así, en el contexto barcelonés, el cambio de paradigma se hizo evidente en cuatro características: (1) el paso de la indiferencia hacia las minorías religiosas a la urgencia de gestionarlas; (2) el cambio de una comunidad imaginada como secular por una comunidad concebida como plural; (3) el paso de un "catolicismo banal" a un catolicismo contestado y (4) el cambio del control a la indiferencia hacia los nuevos movimientos religiosos (Griera, 2012). Además, la posición política y territorial de Barcelona también explica que haya tenido una postura más proactiva que otras urbes en la gestión y acomodación de la diversidad religiosa (Astor et al, 2019). Consecuentemente, en esta ciudad ha habido una voluntad por visibilizar la diversidad religiosa e impulsar políticas inclusivas hacía los cultos minoritarios. En este sentido, cabe destacar que el islam se ha convertido en un objeto de políticas públicas, no sólo por su crecimiento en la esfera pública, sino también por las peculiaridades del modelo catalán de gestión de diversidad religiosa (Garcia-Romeral y Griera, 2011).

En el contexto francés, a pesar de un fuerte centralismo político y administrativo, las ciudades también tienen un papel importante en la gestión del hecho religioso (De Galembert, 2003) que se 
ha visto acrecentado en los últimos años (Martínez-Ariño, 2018). Aspectos como los permisos de construcción de nuevos centros de culto, la gestión de los cementerios comunales o las demandas de menús específicos en los establecimientos escolares son competencias gestionadas por las administraciones locales, convirtiéndose éstas últimas en la "primera puerta" a la que acuden los grupos religiosos para efectuar sus demandas (De Galembert, 2003). En este sentido, si bien la laicidad es un objeto "omnipresente" en los discursos públicos (Bowen, 2007; Liogier, 2006), puede adoptar a su vez formas variadas a nivel local (Martínez-Ariño, 2018). Estas políticas municipales se llevan a cabo en nombre precisamente de la misma laicidad, y pretenden reconocer las minorías religiosas y garantizar los derechos de sus fieles. Sin embargo, la intervención de las administraciones responde también a una voluntad de control sobre los cultos (Fregosi, 2018), y contribuye a definir la legitimidad de las religiones y sus expresiones en el espacio público. Las investigaciones llevadas a cabo en la ciudad de Paris y en sus municipios colindantes muestran así la prevalencia de estas políticas intervencionistas a nivel local, condicionadas a su vez por los límites marcados por el poder central y fuertemente impregnadas por el discurso de la laicidad (Albert Blanco, 2019; Berg, 2019).

\section{Metodología}

Este artículo surge de un proyecto de investigación ${ }^{1}$ más amplio sobre expresiones religiosas en el espacio público que aborda diferentes casos de estudio desde una perspectiva comparada. Con el objetivo de analizar los procesos de (in)visibilización de las expresiones religiosas, los procesos de burocratización y las negociaciones con la sociedad civil, se trata de un estudio de casos múltiple y multisituado, centrado en cinco comunidades religiosas (católicos, sikhs, musulmanes, evangélicos y budistas). En este artículo, sin embargo, nos centramos únicamente en el estudio del islam y, más concretamente, en las expresiones religiosas que protagonizan los musulmanes chiitas en Barcelona y París. Como en el conjunto del proyecto, la metodología utilizada ha sido el enfoque cualitativo, centrado en varias técnicas: la observación de la propia conmemoración de la Ashura de manera continuada durante los años de la investigación, visitas a las comunidades en otros momentos significativos de su vida comunitaria, entrevistas y conversaciones informales. En Barcelona, las observaciones se han realizado siempre de manera colectiva, mientras que en París éstas se han llevado a cabo por un solo miembro del equipo. Las entrevistas se han realizado con actores clave de los dos casos: 7 a líderes y miembros de las comunidades chiitas, 2 a responsables políticos y 4 a técnicos de la administración pública que gestionan directamente la conmemoración de la Ashura. Durante las observaciones también se han tenido conversaciones informales con miembros de las comunidades y técnicos de la administración, que se han incluido como notas en los diarios de campo por su relevancia analítica. También se ha recogido y analizado información historiográfica, periodística y de las redes sociales de las asociaciones chiitas en cuestión.

Concretamente en Barcelona, desde 2015 y hasta 2019, el trabajo de campo ha consistido en la observación y seguimiento de todo el recorrido de la procesión des del oratorio hasta el punto final, donde los participantes realizan un rezo colectivo. También se han observado los rezos y charlas que tenían lugar dentro del oratorio antes de la procesión y se han mantenido numerosas conversaciones informales con participantes en diferentes momentos de la conmemoración. La

\footnotetext{
1 "Expresiones religiosas en el espacio público de Madrid y Barcelona EREUMyB", (CSO2015-66198- P) financiado por el MICINN y llevado a cabo entre los años 2016 y 2019 bajo la dirección de la Dra. Mar Griera (UAB). Los autores agradecen especialmente al Dr. Avi Astor por haber liderado el estudio de caso de la Ashura en Barcelona en el marco del citado proyecto y por haber compartido con nosotros numerosas reflexiones y horas de trabajo de campo en dicha ciudad.
} 
comunidad chií que organiza la Ashura en Barcelona es la asociación Al-Qaim, formada por personas principalmente de origen pakistaní. El oratorio de la comunidad se encuentra en el barrio de Sant Pere, Santa Caterina i la Ribera, que pertenece al distrito de Ciutat Vella, donde la emergencia de la diversidad religiosa ha sido una característica importante en los últimos años ${ }^{2}$. También se han realizado entrevistas con líderes de la comunidad, todos de origen pakistaní salvo un catalán converso, y con diferentes responsables políticos y técnicos de las administraciones públicas implicadas en la regulación de la diversidad religiosa y en la gestión específica de la Ashura.

En París, el trabajo de campo se ha centrado también en la observación, entre 2017 y 2019 , de todas las actividades que tienen lugar el día de la Ashura por parte de dos comunidades situadas en el Departamento de la Seine-Saint-Denis, así como la realización de entrevistas a diferentes miembros jóvenes de estos grupos implicados en los consejos de administración de las comunidades respectivas. Durante las observaciones también se han mantenido conversaciones informales con otros miembros y líderes de las comunidades. Una de estas, llamada Al-Ghadir, aglutina principalmente fieles de origen libanés. Su centro, que acoge tanto la práctica religiosa como otras actividades sociales, se encuentra en Montreuil una ciudad colindante a París, históricamente obrera e industrial, que cuenta con una ingente pluralidad religiosa (Berg, 2019), y sometida hoy en día a un intenso proceso de gentrificación (Collet, 2015). La otra comunidad, Mehfile-Zeinab, se ubica en la ciudad de la Courneuve y reúne sobre todo fieles originarios y descendientes de Madagascar, de las islas Comore y de la Reunión, un colectivo al que se suele llamar "khodja". Para garantizar el anonimato de las personas informantes, hemos obviado datos personales concretos $y$ nombres propios.

Las dos conurbaciones urbanas elegidas para este estudio se caracterizan por presentarse como ciudades globales, cosmopolitas e insertadas en los flujos migratorios (Sassen, 1991), lo que les ha conferido una importante presencia de población musulmana a lo largo de las últimas décadas (Baubérot, 2011; Dargent 2010; Martínez-Ariño et al., 2011). Por otra parte, son ciudades con diferencias en las trayectorias migratorias y con unos marcos legales estatales diferentes. Consideramos que el análisis comparado de los dos casos nos permite mostrar las diferentes lógicas que configuran la Ashura. Además, tal y como hemos explicado en la introducción, partimos de una diferencia clave en como la Ashura toma forma en las dos ciudades: en Barcelona se hacen procesiones en la vía pública mientras que en París la conmemoración se relega al espacio privado. Así, el objetivo del artículo es entender los procesos complejos de gobernanza del hecho religioso que explican esta diferencia. Si bien se podría deducir que estos casos responden a dos modelos contrapuestos, de éxito y fracaso respectivamente, el análisis del conjunto de factores ofrece una mirada mucho más compleja sobre los elementos que se negocian en cada ciudad y juegan un papel determinante en la configuración de la Ashura.

\section{Barcelona y París: dos maneras de conmemorar la Ashura}

Desde el año 2005, la conocida como "Comunidad islámica Al-Qaim", que constituye el principal grupo chií de la ciudad, ha llevado a cabo diferentes manifestaciones en el espacio público (Alonso, 2008) que puede entenderse como una manera de buscar visibilidad y legitimación en un contexto de pluralidad religiosa (Salguero, 2018) Su local, de dimensiones reducidas, está en la calle de San Pere Mitjà, y funciona como mezquita y punto de referencia para la comunidad, donde también desarrolla diferentes actividades sociales y culturales.

\footnotetext{
2 En el Distrito de Ciutat Vella hay 10 oratorios musulmanes, de los cuales sólo uno es chií. En Cataluña, se pueden consultar datos sobre los centros de culto de las minorías religiosas en el "Mapa Religioso de Cataluña", disponible en: http://www.mapareligios.cat/ [consulta: 10 de febrero de 2021].
} 
Hay características del juluus (procesión) que no han variado significativamente a lo largo de los años. El recorrido se inicia en el oratorio de la comunidad donde hombres, mayoritariamente vestidos de negro, comienzan la marcha cargando un objeto que simboliza el féretro del imán Hussain y varias banderas con símbolos y escrituras religiosas. Algunos de los participantes, conocidos como matami, protagonizan un ritual tradicional para recordar el sufrimiento del imán que consiste en una práctica de autoflagelación. Aunque hay diferentes formas de realizar matam (Pinault, 1992), esta práctica la hacen con las manos golpeándose por encima de la camiseta, siguiendo el ritmo marcado por un cántico colectivo. En puntos específicos del itinerario, sobre todo al recorrer las calles más estrechas del barrio, varios hombres con chalecos amarillos aseguran la posibilidad de que los vecinos puedan cruzar las calles, mientras que otros reparten papeles en los que se explica qué es la Ashura y en qué consiste la conmemoración. Mientras todo esto ocurre, se puede observar una gran cantidad de personas, tanto vecinos como miembros de la comunidad, documentando la procesión con móviles y cámaras. Entre estos, cabe destacar también la presencia de medios de comunicación paquistaníes y cámaras de televisión.

La participación de las mujeres se inicia a la mitad del recorrido, donde ellas se incorporan a la procesión de forma discreta. Su entrada no siempre se produce de forma ordenada ni en un punto concreto, sino que a menudo se puede ver cómo se van añadiendo en diferentes puntos de la marcha. A partir del año 2018, la parte de las mujeres estaba dividida por una tela que marcaba la separación con los hombres. No observamos ninguna mujer realizando matam como los hombres, pero algunas se dan golpes flojos siguiendo el mismo ritmo. Siempre siguen el recorrido detrás de ellos, pero al llegar al punto final se vuelven a separar, situándose en un espacio que dividido por vallas cubiertas con telas. Mientras que los hombres empiezan a rezar en un espacio que se puede visualizar fácilmente, las mujeres y los niños se quedan comiendo en una zona más reservada. En todas las procesiones también cabe destacar la presencia de representantes de la administración pública, tanto personal técnico de la Oficina de Asuntos Religiosos como del Distrito de Ciutat Vella. Suelen seguir la procesión de forma discreta, asegurando el buen funcionamiento de ésta, junto a algunas dotaciones de la Guardia Urbana y de los Mossos d'Esquadra, que gestionan el tráfico.

Varios aspectos de la procesión han cambiado significativamente a lo largo de los años, siendo objeto de debate y negociación entre la comunidad y la administración. Después de observar por primera vez una de las conmemoraciones el año 2015, nos dimos cuenta de que el recorrido era muy diferente respecto al que se hizo inicialmente en 2005 , documentado entonces tanto por la prensa como por otra investigación académica sobre rituales islámicos en Cataluña (Alonso, 2008; Moreras et al., 2017). Mientras que esta primera vez la procesión tuvo como punto final la plaza Sant Pere, durante nuestras observaciones desde el año 2015 el itinerario tenía como punto final el Arco de Triunfo (figura 1). Un espacio que, paradójicamente, daba más visibilidad a la conmemoración mientras hacía más fácil un control del espacio a través de vallas y un consecuente "cerco" (Moreras et al., 2017). Sin embargo, en 2018 y en 2019 se volvió a cambiar el recorrido hasta la Estación del Norte y el Mercado del Born respectivamente. En relación con todas estas modificaciones, cabe destacar que la fecha exacta de estos juluus o procesiones es cambiante, debido a que sigue el calendario lunar, y no siempre nos ha sido fácil conocer el día y la hora en que se produciría. Otros cambios significativos que se han producido desde la primera conmemoración han sido relativos al uso de camisetas y a la participación de niños. A raíz de las quejas de vecinos y comerciantes durante los primeros años de la conmemoración el Ayuntamiento de Barcelona estableció como exigencia el uso de camisetas y la prohibición que los menores de 12 años participaran en el ritual. 
[Fig. 1] Rezo colectivo en el paseo del Arco de Triunfo de Barcelona, posterior a la procesión de la Ashura en 2016

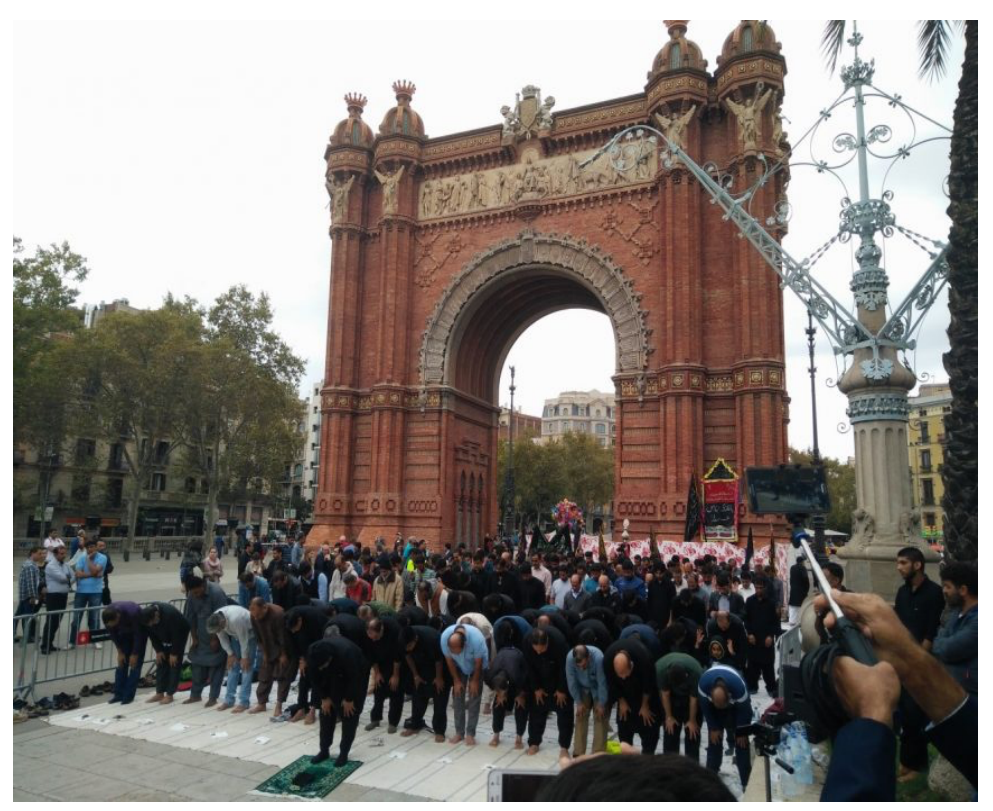

Fuente: Autores

El año 2018 se empezaron a repartir folletos editados por el propio Ayuntamiento de Barcelona. Mientras que la comunidad solía repartir un documento sencillo sin colores ni ilustraciones y con explicaciones que ha menudo tenían errores de traducción, el folleto del Ayuntamiento consiste en una cartulina mucho más visual con logos y explicaciones trabajadas (figura 2). Según nos explicaron algunos de los técnicos de la administración, los folletos fueron resultado de un trabajo conjunto con la comunidad, con quien se consensuaron la información y las imágenes que aparecen. Además, en los folletos se incluye información de festividades religiosas de otras comunidades presentes en el Distrito de Ciutat Vella.

[Fig. 2] Portada del folleto editado por el Ayuntamiento de Barcelona para informar de la Ashura.

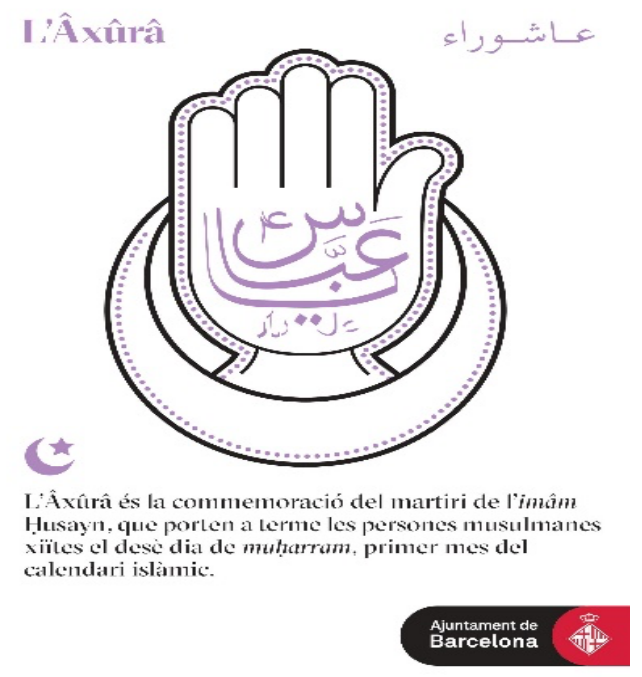

Fuente: Ayuntamiento de Barcelona 
El segundo caso de estudio es el de la ciudad de París, donde existen hoy en día diferentes comunidades chiíes organizadas, principalmente, a partir del origen étnico y/o nacional de sus miembros. A diferencia de Barcelona, las asociaciones chíes parisinas cuentan con un mayor número de fieles, y con locales más grandes y espaciosos. Sin embargo, ninguno de estos se encuentra en el interior de París, sino que se sitúan en ciudades de la periferia. Cabe destacar en este sentido, que una parte importante de estos centros se encuentra en el Departamento 93, llamado de la Seine-Saint-Denis, que se caracteriza por una elevada densidad urbana e indicadores socioeconómicos que se sitúan claramente por debajo de la media nacional y regional (Estèbe, 2018). Las ciudades de este Departamento presentan igualmente una fuerte vitalidad religiosa, especialmente en cuanto al número de asociaciones y centros islámicos (Kepel, 2012).

A diferencia del caso barcelonés, los y las musulmanes chiitas de la región parisina no salen a la calle. Las dos comunidades estudiadas conmemoran la Ashura en el interior de sus locales con un ritual que incluye también la autoflagelación con golpes de mano sobre el pecho acompañados de un canto colectivo ritmado. En el caso de la comunidad Al-Ghadir de Montreuil, son los hombres más jóvenes los que lideran la práctica del matam, haciendo un círculo en el centro de la sala de oraciones de la mezquita. Los otros hombres y las mujeres, éstas en un espacio separado pero visible, se golpean de una manera más suave. Antes, un largo sermón recuerda el sufrimiento y asesinato del imán Hussein, provocando el llanto y las lamentaciones de los asistentes mientras escuchan sentados en el suelo de la sala. Una escena muy similar tiene lugar igualmente en el interior de la comunidad Mehfile Zainab de La Courneuve.

Tanto en Montreuil como en La Courneuve los dos centros islámicos analizados disponen de amplios espacios y de patios donde los fieles se aglutinan y hablan animadamente después del ritual, alrededor de un refrigerio de bebidas y comida dispuesto sobre varias mesas. En estos espacios, que parecen constituir una transición entre el exterior y el interior de la mezquita, se organizan diversos sistemas de control y vigilancia sobre las personas que acceden. Así, para entrar en el local de la comunidad Al-Ghadir hay que pasar por un punto de seguridad en el que tres jóvenes identificados con brazaletes revisan los bolsos y pertenencias de todos los fieles que quieren acceder al centro.

Esta invisibilidad en el espacio público el día de la Ashura, que contrasta tanto con el caso barcelonés como con el de otras ciudades europeas, se intentó revertir el año 2017. En aquella ocasión, algunas decenas de jóvenes musulmanes chiitas de las diferentes comunidades de la región se congregaron en la plaza de la República, en el centro de París. El acto se presentó como una actividad cultural y no religiosa para dar a conocer la figura del imam Hussain, y los jóvenes se limitaban a repartir flores, aguas y chocolatinas acompañadas de folletos explicativos sobre el personaje y la conmemoración de la Ashura (Figura 3). Durante el transcurso de este evento, dos policías de paisano se acercaron a los organizadores para pedirles una copia de la autorización administrativa del acto. Seguidamente, les sugirieron no disgregarse en exceso por la plaza y a adoptar un comportamiento discreto en la repartición de material. 
[Fig 4] Jóvenes de las comunidades chiís de Paris en el acto en la plaza de la República durante la Ashura de 2017

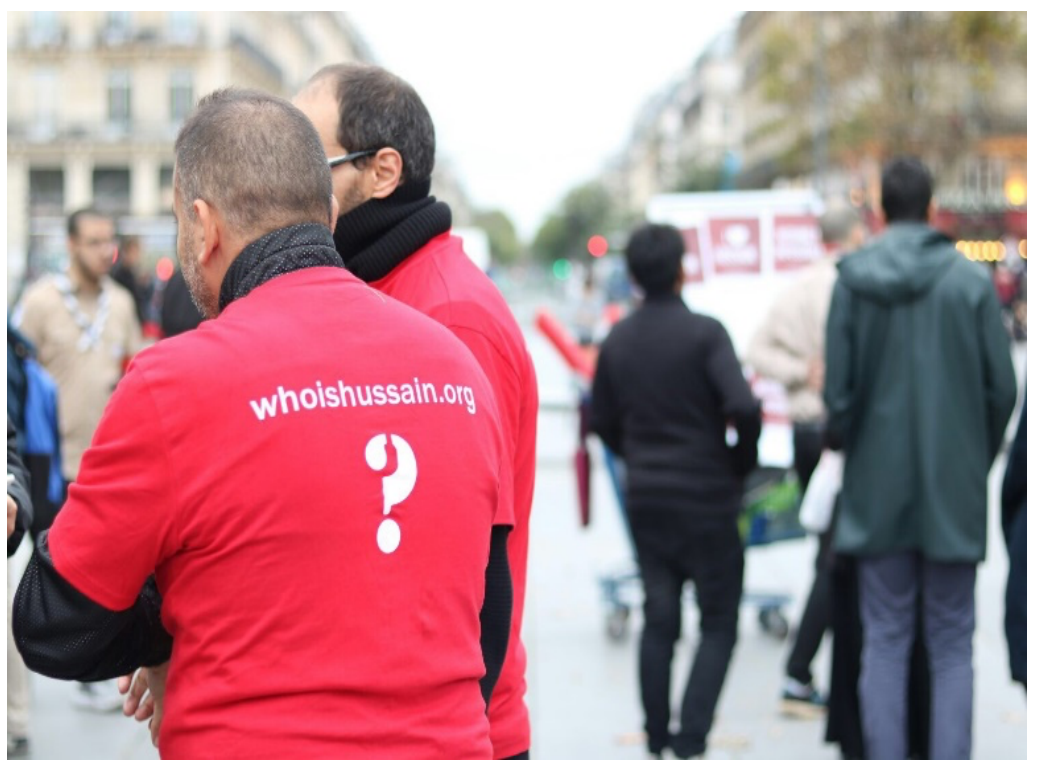

Fuente: "Who is Hussein?-France"

Esta actividad en la plaza de la República se inscribe en una dinámica iniciada en 2014 por un grupo de jóvenes de las diferentes comunidades chiíes de la región. Bajo el paraguas de la organización internacional "Who is Hussain?", estos jóvenes llevan a cabo diversas acciones en el espacio público y los propios centros de culto. Estas iniciativas tienen varios formatos, siendo la mayoría de ellas caritativas o de promoción del diálogo interreligioso. Otras pretenden dar visibilidad al chiismo, como el acto en la plaza de la República o las "vigilias con velas" en la plaza Trocadero, junto a la Torre Eiffel (Figura 4). Estas veladas no han coincidido nunca con el día de la Ashura y, como en el caso del evento en la plaza de la República, se presentan como una actividad "cultural" y no religiosa, una estrategia que se ha identificado también en otras ciudades (Salguero y Hejazi, 2020).

Durante los años que hemos observado la conmemoración de la Ashura en París no hemos podido apreciar cambios significativos. Aparte del acto llevado a cabo en 2017 en la plaza de la República, la conmemoración de la Ashura siempre ha tenido lugar en el interior de los centros de culto. Los testimonios recogidos en entrevistas y conversaciones informales con miembros de las comunidades estudiadas corroboran que los rituales religiosos, como la procesión y la autoflagelación, nunca se han llevado a cabo en el espacio público.

\footnotetext{
3 "Who is Hussain?" es una organización internacional. En su página web se define como un "movimiento por la justicia social" que "se inspira del legado de Hussain". La entidad se constituyó en 2012 en Londres y afirma contar con equipos en 90 ciudades de todo el mundo.
} 
[Fig. 4] Anuncio de una de las veladas organizadas en la plaza Trocadero en 2016.

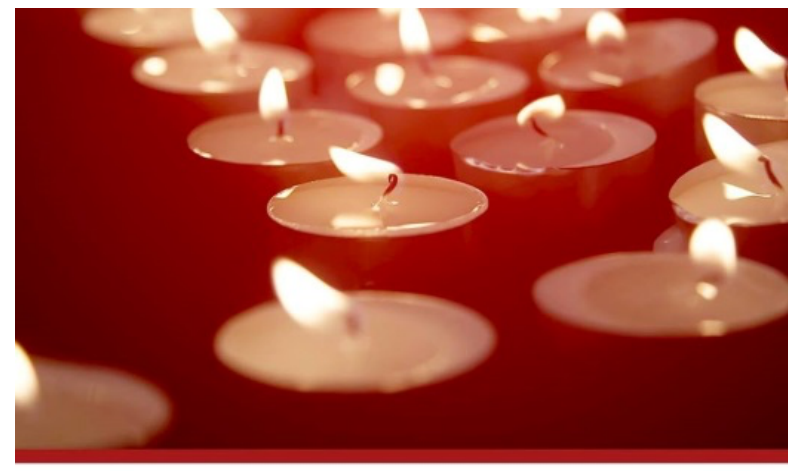

\section{VEILLÉE AUX BOUGIES}

DIMANCHE 27 NOVEMBRE 2016 - 16H30

Parvis des Droits de I'Homme - Place du Trocadéro

\section{whoishussain.org}

Fuente: página Facebook de "Who is Hussein?-France"

\section{Conmemorar la Ashura: un juego de negociaciones y regulaciones.}

Estas dos maneras de conmemorar la Ashura en Barcelona y París, que acabamos de describir en el anterior epígrafe, son claramente el resultado de un juego de negociaciones, regulaciones e innovaciones entre las comunidades y las administraciones públicas de cada ciudad. Los dos casos expuestos evidencian la complejidad de factores que pueden afectar a la práctica y expresión religiosa: la retórica oficial sobre las expresiones religiosas, las normativas locales sobre el uso del espacio público y otros elementos y concepciones sociales que van más allá de la regulación de lo religioso y urbano.

\section{La retórica oficial sobre las expresiones religiosas}

En primer lugar, las retóricas nacionales y locales sobre la libertad religiosa se revelan como elementos clave que configuran la conmemoración de la Ashura en ambas ciudades, siendo recursos simbólicos con implicaciones prácticas y materiales evidentes. En el caso de Barcelona, esta retórica favorece la gestión de la diversidad religiosa y el papel activo de la administración local. Así, la intervención de la administración local se hace principalmente a través de la Oficina de Asuntos Religiosos (OAR) y el Distrito de Ciutat Vella. El objetivo declarado de esta acción municipal es asegurar la libertad de culto y permitir que todas las religiones, especialmente las minoritarias, puedan expresarse en el espacio público en igualdad de condiciones.

En esta línea, cabe destacar la "Medida de gobierno sobre la garantía del trato igualitario a las entidades religiosas en cuanto a la realización de actividades puntuales en el ámbito público", a menudo citada en las entrevistas con los responsables de la administración. Esta medida, promovida por el Ayuntamiento de Barcelona dentro del "Área de Derechos de Ciudadanía, Transparencia y Participación", evidencia claramente la posición del consistorio ante la posibilidad que las 
comunidades religiosas puedan hacer uso del espacio público. De hecho, en la norma se reconocen dos supuestos que se aplican claramente al caso de la Ashura:

"El uso del espacio público por parte de entidades religiosas se puede producir para la celebración de actos de culto al aire libre, sobre todo en aquellos casos en que se reúne un número excepcional de personas que se acercan de otros territorios. Otro supuesto es el de las actividades que consisten en un recorrido por la vía pública (procesiones, etc.)" Traducción de los autores. (Ayuntamiento de Barcelona: 14).

Así, en este documento el Ayuntamiento deja claro que su gestión de la diversidad religiosa parte del marco jurídico vigente relativo a la libertad de culto y a la Declaración Universal de Derechos Humanos. Entre sus objetivos, se pueden encontrar "normalizar la presencia de la diversidad religiosa en la ciudad', así como "trabajar para mejorar el acceso con carácter puntual a equipamientos y vía pública" Traducción de los autores. (Ayuntamiento de Barcelona, 2016: 20).

Por tanto, el papel de la administración es clave para entender la posibilidad que la Ashura tenga lugar en las calles de Barcelona. Desde la OAR se ofrece un acompañamiento para las gestiones burocráticas y la redacción de permisos, mientras que también se convocan reuniones o visitas para ultimar detalles logísticos. Además, tal y como se ha explicado en la descripción etnográfica, la presencia de personal de la administración durante la Ashura es constante a lo largo de los años. Según nos explicó la consejera del Distrito de Ciutat Vella ${ }^{4}$ responsable de los temas de "interculturalidad" durante el mandato 2015-2019, este acompañamiento se reforzó después de la publicación de la Medida de Gobierno y también incluyó formación para el personal de atención de los equipamientos y los técnicos de distrito, para que tengan en cuenta los objetivos de la "Medida" en el desempeño de sus funciones. Esta responsable política también destacó que se ha hecho una labor intensa para que las gestiones burocráticas se acomoden a los ritmos de las celebraciones. Una de las particularidades de la Ashura es que hasta pocos días antes no se conoce la fecha exacta de su conmemoración, ya que depende del ciclo lunar y el día no siempre coincide con una fecha exacta del calendario solar.

En este sentido, la consejera explicó que para garantizar que se puedan celebrar estos actos, se ha ido interiorizando que es necesario hacer reservas de equipamientos o vía pública con un margen de dos días. Así, cuando se concreta la fecha exacta, que suele ser unos días antes, los espacios están asegurados. Aunque destacó que ha sido un trabajo que a nivel interno de la administración "ha costado", explicó que ha sido resultado del impulso político y de mucha perseverancia (entrevista EB_01, consejera de distrito de Ciutat Vella).

El personal técnico del Ayuntamiento también juega un papel importante de intermediación con los Mossos d'Esquadra y la Guardia urbana. La presencia de estos efectivos se justifica ante la necesidad de garantizar la movilidad y la seguridad. De hecho, el permiso que deben solicitar para poder hacer la procesión es un formulario bastante completo con el que se garantiza que los cuerpos de seguridad tendrán los detalles del evento (Modelo FE0 $1^{5}$ ). Además, cuando ha habido algún problema con la Guardia Urbana, o ésta no ha llegado a la hora, el personal de la OAR reclama su presencia y hace de intermediario.

\footnotetext{
${ }^{4}$ Los consejeros/as de los distritos de Barcelona son cargos políticos. Se eligen proporcionalmente de manera indirecta en función de los resultados de cada partido en las elecciones municipales, y los que pertenecen a la mayoría de gobierno asisten al concejal de distrito en temas concretos.

${ }^{5}$ Este modelo consiste en la "Solicitud de licencia de espectáculos públicos y actividades de carácter extraordinario organizados en la vía pública hasta 1000 personas de aforo (sin pirotecnia o con pirotecnia <10Kg))", disponible en: https://w9.bcn.cat/tramits/SSTT_VP/FE01_XXPB.pdf [consulta: 28/11/2019].
} 
El papel de la administración también es muy importante de cara a la comunidad y las reuniones que pueden tener para negociar los detalles de la conmemoración. En las entrevistas con el personal técnico de la administración, se destaca especialmente la disposición de los líderes de la comunidad para entender y gestionar las propuestas que se hacen. Reconocen que ellos también ponen sus límites como comunidad, pero señalan su predisposición a encontrar soluciones para poder llevar a cabo el juluus. Además, en esta línea también hay que tener en cuenta que a la Ashura pueden asistir personas de diferentes zonas de Barcelona y de fuera de la ciudad y del país (Astor et.al, 2018). Esto explica que, en muchos casos, desde la comunidad quieran llegar a acuerdos que puedan satisfacer a todos sus miembros.

Finalmente, la introducción reciente de unos folletos mencionados más arriba sobre la Ashura, editados por el Ayuntamiento de Barcelona, es otro elemento que refuerza y materializa la retórica local sobre la diversidad religiosa. Uno de los objetivos de estos materiales es poder ofrecer información a vecinos y comerciantes y relajar posibles conflictos. Además, como estos documentos también incluyen información de otras festividades religiosas, permiten dar a conocer la diversidad religiosa del barrio y del distrito. Desde la administración han destacado que es una iniciativa que se ha trabajado juntamente con la comunidad y que la valoración es muy positiva:

"yo creo que hay un punto de que en todo el proceso sintieron como que se ponía en valor, de alguna manera, su cultura y su tradición, ellos se sentían como bastante honrados para decir, "estoy pudiendo comunicar algo que para mí es súper importante", desde lo local" (entrevista EB_02, técnico de la administración)

En el caso de París, es otra retórica la que determina en parte la forma que adopta la Ashura. En este sentido, el modelo francés de gestión de cultos, la llamada "laicidad" (Baubérot, 2017), parece actuar como un freno simbólico a la conmemoración de la Ashura en el espacio público. A pesar de que nada impide formalmente la celebración de este tipo de actividades, la laicidad es percibida por nuestros informantes como un marco limitador de ciertas manifestaciones religiosas, una concepción que puede estar condicionada por los debates recurrentes sobre el islam en el contexto francés y que han contribuido a erigirlo en un "problema público" (Hajjat et Mohammed, 2013)

Cuando preguntamos a un joven miembro de la comunidad Al Ghadir de Montreuil por qué no salen de la mezquita, no dudó en afirmar que "hay que tener las autorizaciones, no es fácil", para añadir que, "deberíamos probar, pero yo creo que sería difícil. Sí, es difícil en Francia. De hecho, los símbolos religiosos en un espacio público que debe ser laico ..." (entrevista EP_01, comunidad chií). Siguiendo esta línea, otro joven de la misma asociación nos comentaba durante el acto en la plaza de la República en octubre de 2017 que, precisamente, organizaban esta actividad para "romper prejuicios", ya que "en Francia no es fácil"(diario de campo, P_01/10/2017). Del mismo modo, un informante de la comunidad Mehfile-Zeinab de La Courneuve señalaba que la sociedad francesa "no está preparada" para ver este tipo de manifestaciones religiosas en el espacio público (diario de campo, P_20/11/2018).

La laicidad adopta así la forma de un objeto "omnipresente" (Bowen, 2007), que no sólo orienta la acción de los poderes públicos, sino que adquiere una potencia discursiva que parece desincentivar la conmemoración de la Ashura en el espacio público. La retórica sobre la laicidad se acompaña de una supuesta voluntad de respeto a la libertad de culto, pero también por mecanismos de 
protección, control y vigilancia sobre las prácticas religiosas. Como adelantábamos anteriormente, la laicidad francesa no se guía por un principio de neutralidad, sino por la intervención activa del Estado en la definición de los cultos legítimos y de las manifestaciones que se pueden llevar a cabo en el espacio público (Liogier, 2006). Así, y a pesar del centralismo político y administrativo de Francia, los ayuntamientos también tienen un papel importante en la definición de la laicidad y la forma que ésta puede adoptar a nivel local (Martínez-Ariño, 2018).

En este sentido, hay que señalar que a pesar de no conmemorar la Ashura en el espacio público, las comunidades chiitas de la región parisina tienen una interlocución estable y directa con las administraciones que se enmarca en este modelo multiforme y punzante que es la laicidad francesa. Así, por ejemplo, una placa colocada en la entrada del centro Mehfile-Zeinab de la Courneuve recuerda que el espacio fue inaugurado por "el alcalde-senador" del municipio. Del mismo modo, el presidente de esta comunidad nos explicaba que unas semanas antes de la Ashura del año 2018 había mantenido una reunión con representantes de las administraciones con el fin de explicarles el programa y las actividades previstas en la conmemoración (diario de campo, P_20/09/2018).

Estos contactos, que son habituales entre las comunidades musulmanas y los poderes públicos franceses, parecen tener una doble vertiente. Por un lado, constituyen una forma de fiscalización de la práctica religiosa, para que ésta quede conforme a la laicidad: discreta y relegada en un espacio cerrado. Por el otro, también parecen constituir un momento en el que las comunidades plantean y negocian sus necesidades en términos de protección y acompañamiento por parte de las administraciones y fuerzas de seguridad. En este sentido, cuando un líder de la asociación MehfileZeinab nos relataba estos encuentros afirmaba "ser consciente de que no podemos disponer de un policía ante la puerta las 24 horas del día" (diario de campo, P_20/09/2018). Se refería así a la necesidad de protección en un contexto de tensión provocado por las acciones de Estado islámico. Asimismo, en esta relación con las autoridades, la comunidad ofrece promover, en palabras de su presidente, "un islam moderado" (diario de campo, P_20/09/2018).

Tanto en París como en Barcelona, lo que aquí hemos llamado "retórica oficial sobre las expresiones religiosas" tiene una carga política importante. Efectivamente, la variable ideológica del poder (en este caso el local) no es indiferente, aunque la perspectiva comparada deja entrever una relación de los entes municipales con el hecho religioso que va más allá del eje izquierda-derecha. En Barcelona, la Oficina de Asuntos Religiosos (OAR) se impulsa bajo un gobierno liderado por el Partido Socialista, en un momento (2004) en el que las élites locales quieren consolidar la proyección internacional de la ciudad y su carácter "cosmopolita".

El breve periodo de gobierno nacionalista (2011-2015) no altera el funcionamiento de la OAR, aunque la llegada al poder de Barcelona en Comú en 2015 parece dar un nuevo impulso al tratamiento de la cuestión religiosa con la aprobación de la Medida antes citada o la adopción de un Plan municipal contra la islamofobia (López-Bargados, 2018). Las dos ciudades colindantes de París que acogen las comunidades chiíes aquí mencionadas son por su parte bastiones históricos de la izquierda. Ambas han sido gobernadas desde hace décadas por el Partido comunista, formando parte de lo que comúnmente se ha identificado como el "cinturón rojo" de la capital francesa. Sin embargo, en el caso de Montreuil se produjo en 2008 un vuelco electoral en beneficio de los ecologistas, que gobernaron la ciudad hasta 2013 cuando nuevamente pasó a manos de una coalición que integraba los comunistas. Tal como sugiere Berg (2019), estos cambios en la alcaldía de Montreuil han tenido consecuencias sobre la gobernanza del hecho religioso, dando lugar a políticas que priorizan estrategias distintas en función del momento y de los actores. Paradójicamente, todas ellas se apoyan en la laicidad, reforzando la unanimidad discursiva y la vertiente intervencionista de este principio expuesta anteriormente. 
Las normativas del espacio público en la intersección de la expresión religiosa

En segundo lugar, hay que tener en cuenta que las manifestaciones religiosas como la Ashura también pueden verse afectadas por las normativas locales relativas al uso del espacio urbano. En Barcelona, la Medida de Gobierno antes citada reconoce que se producen problemas en el acceso al espacio público, sobre todo derivados de la saturación de ciertos lugares del Distrito de Ciutat Vella, donde hay mucha densidad de población, un número elevado de entidades y una importante presión turística. En el caso de la Ashura, hay que tener en cuenta diferentes normativas a la hora de decidir el recorrido de la procesión.

Una de las normativas que pueden afectar a la conmemoración de la Ashura es la de la sonorización. En 2018, se modificó el recorrido del juluus porque tenía lugar durante el verano y en Ciutat Vella se había establecido una normativa que impedía hacer ruido amplificado a partir de las $20 \mathrm{~h}$ de lunes a jueves. Así, según nos explicaron los técnicos de la administración, se pactó un cambio en el itinerario de la procesión teniendo en cuenta que la normativa de sonorización del Distrito del Eixample no contemplaba esta limitación, y se estableció la Estación del Norte como punto final del recorrido al encontrarse en otro distrito. Sin embargo, este cambio implicó la coordinación de responsables de dos distritos, ya que la procesión comenzaba en Ciutat Vella pero terminaba en el Eixample, suponiendo la duplicación de permisos y gestiones.

En el caso de Barcelona, también cabe destacar que el año 2006 se aprobó una ordenanza de civismo que entre otras cuestiones prohibía pasear por la calle sin camiseta. El principal argumento expuesto por los responsables políticos a la hora de adoptar esta medida se construyó a partir de lo que se percibía como una práctica "incívica", protagonizada principalmente por turistas al ir o venir de las playas de la ciudad. Siendo algo que pretendía regular el día a día de Barcelona, supuso una limitación directa de una característica propia del ritual de la Ashura. Bajo esta normativa, los hombres que efectúan el matam deben llevar el torso cubierto durante todo el recorrido. Por un lado, la mayoría del personal técnico con el que hemos podido hablar apunta que esta normativa no supone ningún problema con la comunidad. Sin embargo, algunos de sus líderes nos han hecho referencias a otras ciudades europeas en las que el matam sí puede llevarse a cabo sin camiseta. Además, parece ser que en algunas reuniones con el personal de la administración los líderes mostraron vídeos de estos eventos para demostrar que es posible en otros lugares. A pesar de que no es una petición clave e imprescindible, los dirigentes de la comunidad son conscientes de que están limitados de una forma muy específica en el contexto barcelonés.

En el caso francés, la autorización para la realización de cualquier tipo de manifestación cultural, política o religiosa en el espacio público es responsabilidad de las subdelegaciones del gobierno central en cada Departamento, las llamadas prefecturas. En este sentido, los miembros de las comunidades chiíes con los que pudimos hablar se refieren a los "permisos" que habría que pedir ante estas instancias para poder conmemorar la Ashura, insinuando un proceso burocrático percibido como difícil y complejo, condicionado además por el discurso oficial sobre la laicidad.

Por otra parte, la autorización de determinados eventos en el espacio público queda sometida al contexto político de cada momento. Cabe destacar así la aplicación del estado de urgencia tras los atentados de 2015, una situación que otorgaba aún más prerrogativas a las prefecturas a la hora de permitir o no determinadas actividades. En este sentido, numerosas manifestaciones sindicales o culturales se vieron afectadas por esta medida, siendo modificados sus emplazamientos e 
itinerarios. Esta situación parece que afectó igualmente las comunidades chíes. Algunos informantes nos contaron que en 2015 habían previsto una velada con velas el día 15 de noviembre. Dos días antes, el 13 de noviembre, varios establecimientos de ocio de Paris fueron objeto de un ataque terrorista que causó decenas de muertos. En relación a este contexto, una chica de la comunidad Mehfile-Zeinab nos explicó que el acto no se pudo hacer "debido a los atentados" (diario de campo, P_01/10/2017). Una publicación de aquella fecha en las redes sociales de la plataforma "Who is Hussain?" lo corroboraba explicando que "después de los acontecimientos espantosos" y "por motivos de seguridad", la velada quedaba pospuesta (página Facebook de "Who is Hussain?France").

Más allá de los contextos temporales particulares, como la situación derivada de los atentados de 2015, las actividades realizadas en el espacio público para las comunidades chiíes parisinas parecen adaptarse también a los usos sociales aceptados y autorizados de determinados espacios urbanos. Así, no deja de ser significativo que los emplazamientos escogidos para la celebración de estos actos sean la plaza de la República y la de Trocadero. La primera es conocida por ser el punto de inicio o final de numerosas manifestaciones políticas y sindicales, así como para acoger actividades culturales y conciertos. Es habitual que en la plaza coincidan en un mismo día diversas actividades o concentraciones, y que éstas se distribuyan en diferentes puntos. Por su parte, la plaza de Trocadero es un emplazamiento turístico, ya que es un punto de paso casi obligado para llegar a la Torre Eiffel o para fotografiarla. Este espacio no suele acoger grandes manifestaciones políticas, pero sí concentraciones más reducidas con el objetivo de visibilizar y denunciar ciertas situaciones de conflicto o de vulneración de derechos humanos en Francia o en todo el mundo.

La elección de estos espacios por parte de los jóvenes chiíes de la región parisina representa, en primer lugar, un intento de apropiarse temporalmente de dos plazas con una fuerte carga simbólica en el imaginario nacional francés. Como muestra L. Endelstein (2017) para el caso de la celebración judía de la Hanukkah en estos mismos espacios, se canaliza así la reivindicación de una doble pertenencia: a la nación, pero también a una comunidad religiosa transnacional. Los actos organizados por las comunidades chíes en estos dos espacios parecen adaptarse así a las actividades que llevan a cabo otros grupos políticos, sindicales o asociativos. En este sentido, las observaciones llevadas a cabo en la plaza de la República, pero también las informaciones y fotografías de las vigilias en Trocadero en las redes sociales, muestran un repertorio variado de acciones que también podrían llevar a cabo grupos seculares. Mesas informativas, reparto de hojas explicativas, pancartas y carteles pidiendo "fraternidad" o discursos sobre "la paz en el mundo" constituyen una puesta en escena que ejemplifica una frontera porosa entre las dimensiones secular y religiosa. Un joven de la comunidad Al-Ghadir de Montreuil, definía así la actividad llevada a cabo en la plaza de la República:

“El evento que hicieron por ejemplo en la plaza de la República era otro tipo de actividad. Más correcta, seguramente más aceptable por el entorno. Sobre todo, en Francia, donde es un poco complicado y tienen problemas para aceptar las religiones y es realmente laica" (entrevista EP_01, comunidad chií)

La estrategia de las comunidades chíes parisinas para salir al espacio público pasa por una adaptación de las manifestaciones religiosas favoreciendo unas expresiones más "correctas" en un contexto particular (Martínez-Ariño y Griera, 2020). Esta concepción está evidentemente influenciada por la retórica sobre la laicidad mencionada anteriormente, a la vez que se adapta y toma forma en función de los usos esperados y "aceptables" de espacios urbanos concretos.

Por otra parte, la cuestión del género también está presente en las negociaciones de la Ashura como parte de lo que se espera de la participación en el espacio público. Esto es evidente sobre todo en 
el caso de Barcelona, donde para algunos técnicos la participación igualitaria de hombres y mujeres en la procesión es una cuestión sensible y relevante. De hecho, en la medida de gobierno se especifica que únicamente se van a promover las actividades que garanticen la igualdad de género. Así, algunos técnicos de la administración explicaron que en varias reuniones se ha intentado fomentar que hombres y mujeres compartieran más espacios, o al menos que no se tuviese que separar físicamente el espacio donde están las mujeres en el punto final de la procesión. Sin embargo, otras personas de la administración destacaron positivamente que haya un lugar reservado para las mujeres y que se garantice su participación, aunque sea de forma diferenciada.

En el caso de París, la participación de las mujeres parece no suscitar ninguna tensión relevante por el hecho que, como se ha señalado anteriormente, el ritual religioso no se realiza en el espacio público. Sin embargo, durante el evento "cultural" llevado a cabo en la plaza de la República, esta cuestión emergió en algunas discusiones entre los jóvenes chiitas y los transeúntes, mostrando el peso que los debates sobre islam y género tienen en Francia (Bowen, 2007; Gole, 2015). En este sentido, uno de los organizadores nos explicó que algunos pasantes habían hecho comentarios negativos relativos a la vestimenta y al velo de las participantes, así como sobre la percepción de un uso segregado del espacio público por parte de hombres y mujeres. Un informante insistió en desmentir este último supuesto, afirmando que chicos y chicas participaban todos juntos y "mezclados" en el desarrollo de la actividad.

\section{Un calendario marcado por elementos sociales mas allá del hecho religioso y urbano}

Finalmente, la Ashura está condicionada por las concepciones sociales sobre el hecho religioso, pero también por otros elementos aparentemente neutros que pueden tener un impacto directo y determinante. Esto es especialmente evidente cuando tenemos en cuenta el efecto del calendario de celebraciones. La Ashura tiene lugar en el décimo día del Muharram, el primer mes del calendario islámico. Éste sigue el calendario lunar por lo que no se corresponde con el calendario oficial, que sigue el ciclo solar. Así, tal y como sucede con otras fechas relevantes para los musulmanes y otras confesiones religiosas, la Ashura se conmemora en una fecha diferente cada año. En contextos diaspóricos, donde las comunidades musulmanas siguen siendo minoritarias, estas fechas no son conocidas ni reconocidas en el calendario hegemónico. Consecuentemente, la Ashura puede coincidir y competir con otras festividades que sí son reconocidas en el calendario oficial y que, además, pueden tener una connotación religiosa.

En el caso barcelonés, a pesar del carácter aconfesional del Estado, el calendario de celebraciones estatales tiene presente festividades con una carga religiosa muy evidente como la Semana Santa y la Navidad. Además, también reconoce otras festividades con un carácter religioso menos evidente a nivel local. Por ejemplo, el 24 de septiembre, el día de la Virgen de la Mercè, patrona de Barcelona, se convierte en un día festivo para todos los vecinos y vecinas con una importante oferta cultural y musical en la ciudad. En el año 2018, la Ashura debía conmemorarse el 20 de septiembre. Ese día, mientras se estaba preparando el escenario para los conciertos de "La Mercè", que tendrían lugar durante varios días en el paseo donde se encuentra el Arco de Triunfo, los miembros de la comunidad de Al-Qaim tuvieron que modificar su recorrido y hacer un camino más largo hasta el Mercado del Born. Uno de los efectos principales de este cambio de itinerario fue, según la comunidad, la pérdida de visibilidad en relación con el barrio. Uno de sus líderes nos comentó:

"La zona esa del Arco del Triunfo era la zona de relaciones del barrio y cuando estos llegaron pues era la zona de visibilidad, de relaciones del barrio y claro, ir al Arco del 
Triunfo y ser capaces de estar en el Arco del Triunfo era, "que nos vean" (entrevista EB_03, comunidad chií).

Mientras que el reconocimiento de festividades con connotación católica no se problematiza por su justificación bajo la idea de "tradición" o "herencia cultural" (Astor et al., 2017) y por las consecuencias del "ambient faith" (Engelke, 2012), se crea una situación de privilegio ante las fechas de las celebraciones de otras minorías. Es cierto que, tal y como argumenta Griera (2012), la conmemoración de la Ashura y la consiguiente normalización de la diversidad religiosa puede suponer un "catolicismo contestado" y poner en duda los privilegios de las festividades y el calendario católico. Sin embargo, hay que tener en cuenta que en el caso de la Ashura el "catolicismo banal" (Griera, 2012; Griera y Clot, 2015) condiciona directamente la conmemoración y las características del juluus, ya que las fechas no son conocidas ni reconocidas. Consecuentemente, mientras que hay un intento de visibilizar la pluralidad religiosa, las festividades de la comunidad musulmana chií están sometidas a la priorización social y administrativa de otras celebraciones.

Siguiendo esta misma línea, también hay que destacar lo que sucedió en 2016 y 2017 . En el año 2016 la Ashura fue el 12 de octubre, un día donde había muchas manifestaciones convocadas a raíz tanto de la celebración como del rechazo del "Día de la Hispanidad". Aunque esto no afectó al recorrido, la procesión tuvo que llevarse a cabo sin todos los efectivos de la Guardia Urbana, ya que éstos estaban gestionando las citadas manifestaciones. En el año 2017, y como se ha mencionado en la introducción, la Ashura debía conmemorarse el 1 de octubre, el mismo día en que había convocado un referéndum sobre la independencia de Cataluña. Como días antes ya se preveía que podría ser una jornada conflictiva, el personal técnico del Ayuntamiento estuvo en contacto con los líderes de la comunidad para avisarles de que la situación se podría complicar. Un día antes acabaron decidiendo que la procesión en la vía pública no sería posible y habilitaron un equipamiento cerrado en el barrio del Raval. Aunque estos casos no están tan relacionados con festividades católicas, evidencian y remarcan la condición minoritaria de las festividades chiitas. Mientras que sería difícil convocar una manifestación o una cita política en otra fecha como el día de Navidad o durante la Semana Santa, la conmemoración de la Ashura queda escondida en el calendario.

La persistencia de esta forma de "catolicismo banal" no es exclusiva del caso barcelonés. Así, y a pesar de la retórica sobre la laicidad, numerosos ejemplos muestran que en Francia el catolicismo preserva igualmente una condición de normalidad, bajo la apariencia de tradición cultural, que no comparte con las otras religiones. En este sentido, el calendario de festividades también se estructura en torno a celebraciones de referencia católica: del total de días festivos reconocidos oficialmente, siete son de raíz cristiana. Desde hace algunos años, las administraciones pueden autorizar una "ausencia" para sus trabajadores con ocasión de las grandes festividades de cada religión. Este dispositivo resta, sin embargo, limitado a los empleados de la función pública y tiende a privilegiar aquellas fechas más conocidas y aceptadas socialmente, como el Eid-al-adha para los musulmanes o el Yom Kippur para los judíos (Fornerod, 2019). En el caso parisino el calendario afecta las prácticas y la organización de los musulmanes chiíes cuando la Ashura coincide con un día laborable. Como nos comentaba un joven miembro de la comunidad Al-Ghadir, cuando esto sucede los fieles piden fiesta a sus puestos de trabajo Esto no siempre es posible y acuden entonces a la mezquita en el momento que pueden. A diferencia de Barcelona, relegar la conmemoración en el interior de los centros de culto hace que el calendario no tenga implicaciones en la utilización del espacio público. 


\section{Conclusiones}

A pesar de la retórica y de las políticas de reconocimiento de las minorías religiosas en las grandes ciudades europeas, el análisis de la Ashura en contextos diaspóricos evidencia los diferentes tipos de limitaciones externas que influyen en la manera que las comunidades religiosas pueden hacer uso del espacio público. Los dos casos de estudio presentados muestran unas lógicas complejas de regulación, acompañamiento y control de la expresión y la práctica religiosa. Además, confirman la importancia de considerar las ciudades como "espacios políticos" (Martinez-Ariño, 2018).

Con el análisis de la Ashura hemos querido destacar los procesos complejos de negociación y regulación de las expresiones religiosas. La forma de conmemorar la Ashura en Barcelona no sólo es resultado de una retórica que favorece el uso del espacio bajo la idea de la promoción de la diversidad religiosa, como tampoco lo es en Paris como consecuencia de una regulación sujeta a la laicidad. Tal y como hemos querido mostrar en la discusión de los resultados, la Ashura está condicionada por muchos otros factores que son menos evidentes y que a veces pueden parecer contradictorios con los discursos y políticas que pretenden favorecer la diversidad religiosa.

Aunque en Barcelona hay un impulso relevante por parte de las administraciones para promover la posibilidad de utilizar el espacio público por parte de las comunidades religiosas, visibilizando así la pluralidad religiosa que caracteriza la ciudad, las normativas sobre el uso del espacio urbano y el calendario hegemónico de las festividades locales y nacionales tienen un efecto directo en cómo la comunidad chií de Barcelona puede llevar a cabo la procesión. En el caso de París, no es tanto la regulación local la que marca la forma de la Ashura sino la retórica construida alrededor de la laicidad francesa. A pesar de que no hay normativas explícitas que impidan las conmemoraciones religiosas en el espacio público, las comunidades chiitas parecen interiorizar una limitación simbólica sobre el uso de este. Asimismo, las comunidades adoptan estrategias que les permiten ser visibles a través de actos y eventos presentados como "culturales" y que responden, además, a los usos esperados y aceptados los espacios urbanos. 


\section{Referencias}

AJUNTAMENT DE BARCELONA (2016): Mesura de Govern sobre la garantia del tracte igualitari a les entitats religioses quant a la realització d'activitats puntuals a l'àmbit públic, disponible en: https://ajuntament.barcelona.cat/bcnacciointercultural/sites/default/files/documentos/mesura t racte igualitari entitats religioses cat1.pdf [consulta: 28 de noviembre de 2019].

ALBERT BLANCO, Víctor (2019): “Valoriser le quartier par la diversité religieuse: regards crosiés entre la Goutte d'Or (Paris) et le Raval (Barcelone)", Cahiers de Géographie du Québec, vol. 63, no 178, pp. 21-35.

ALLIEVI, Stephano (2009): Conflicts over mosques in Europe. Policy issues and trends, London, Alliance Publishing Test.

ALONSO, Marta (2008): “Chí́tas, ashura y espacio público barcelonés: de los callejones a la Avenida", en Y. Mellado (coord.), La dinámica del contacto. Movilidad, encuentro y conflicto en las relaciones interculturales, Barcelona: $\mathrm{CIDOB}, \mathrm{pp} .23-32$.

ASTOR, Avi (2017): Rebuilding Islam in Contemporary Spain: The Politics of Mosque Establishment, 1976-2013, Eastbourne, Sussex Academic Press. DOI: https://doi.org/ $\underline{10.1080 / 09518967.2018 .1535403}$

ASTOR, Avi; ALBERT BLANCO, Víctor y MARTÍNEZ CUADROS, Rosa (2018): “The Politics of 'Tradition' and the Production of Diasporic Shia Religiosity", POMEPS Studies, 32: The Politics of Islam in Europe and North America, disponible en https://pomeps.org/2018/12/18/the-politics-of-tradition-andthe-production-of- diasporic-shia-religiosity/ [consulta: 14 de marzo de 2019].

ASTOR, Avi; BURCHARDT, Marian y GRIERA, Mar (2017): “The politics of religious heritage: framing claims to religion as culture in Spain", Journal for the Scientific Study of Religion, 56(1), pp. 126-142. DOI: https://doi.org/10.1111/jssr.12321

ASTOR, Astor; GRIERA, Mar, y CORNEJO, Mónica (2019): "Religious governance in the Spanish city: hands-on versus hands-off approaches to accommodating religious diversity in Barcelona and Madrid", Religion, State \& Society, 47(4-5), pp. 390-404. DOI: https://doi.org/10.1080/09637494.2019.1668213

BAUBEROT, Jena (2011): “ Ville et religion : Dieu change à Paris », en Michel Wieviorka (ed.) La ville, Auxerre, Éditions Sciences Humaines, pp. 237-251.

BAUBEROT, Jean (2017) : Histoire de la laïcité en France, Paris, Presses Universitaires de France. DOI : https://doi.org/10.1484/M.BEHE-EB.5.113083

BECCI, Irene; BURCHARD, Marian y CASANOVA, José (2013): Topographies of faith. Religion in Urban Spaces, Boston, Brill. DOI: https://doi.org/10.1163/9789004249073

BECCI, Irene; BURCHARDT, Marian y GIORDA, Maria-Chiara (2017): "Religious super-diversity and spatial strategies in two European cities", Current Sociology, 65 (1), pp. 73-91. DOI: https://doi.org/10.1177/0011392116632030

BECKFORD, James (2003): Social theory and religion, Cambridge, Cambridge University Press. DOI: https://doi.org/10.1017/CBO9780511520754 
V. Albert Blanco y R. Martínez Cuadros, Conmemorar la Ashura en la diáspora ...

BERG, Anna Lea (2019): "From religious secular place-making: How does the secular matter for religious place construction in the local?", Social Compass, 66 (1), pp. 35-48. DOI: https://doi.org/10.1177/0037768618813774

BOWEN, John (2007): Why the French Don't Like Headscarves: Islam, the State, and Public Space, Princeton, Princeton University Press. DOI: https://doi.org/10.1515/9781400837564

CASANOVA, José (2011): Public religions in the modern world, Chicago, University of Chicago Press.

CESARI, Jocelyne (2003): "Muslim minorities in Europe: the silent revolution", en J. ESPOSITO, John y BURGAT, François (eds.) Modernising Islam: Religion in the Public Sphere in the Middle East and in Europe, London, Hurst, pp. 251-269.

CESARI, Jocelyne (2004): L'islam à l'épreuve de l'Occident, Paris, La Découverte.

COLLET, Anaïs (2015): Rester bourgeois. Les quartiers populaires, nouveaux chantiers de la distinction, Paris, La Découverte.

DARGENT, Claude (2010): " La population musulmane de France : de l'ombre à la lumière ? », Revue française de sociologie, vol. 51(2), pp. 219-246. DOI : https://doi.org/10.3917/rfs.512.0219

DE GALEMBERT, Claire (2003): “La gestion publique de I'islam en France et en Allemagne: De l'improvisation de pratiques in situ à l'amorce d'un processus de régulation nationale", Revue internationale et stratégique, 52(4), pp. 67-78. DOI : https://doi.org/10.3917/ris.052.0067

DUDERIJA, Adis. (2007): “Literature Review: Identity Construction in the Context of Being a Minority Immigrant Religion: The Case of Western-born Muslims", Immigrants \& Minorities, 25(2), pp. 141162. DOI: https://doi.org/10.1080/02619280802018132

ENDELSTEIN, Lucine (2017): “Lumières sur la ville”, Archives de sciences sociales des religions, 177, pp. 51-71. DOI : https://doi.org/10.4000/assr.29252

ENGELKE, Matthew (2012): "Angels in Swindon: Public religion and ambient faith in England", American Ethnologist, 39(1), pp. 155-170. DOI: https://doi.org/10.1111/j.1548-1425.2011.01355.x

ESTÈBE, Philippe (2018): “Seine-Saint-Denis : le sas et la nasse”, Tous urbains, 22(2), pp. 34-43. DOI : https://doi.org/10.3917/tu.022.0034

ESTRUCH, Joan, GÓMEZ, Joan, GRIERA, Mar y IGLESIAS, Agustí (2004): Les altres religions. Minories religioses a Catalunya. Barcelona, Ed. Mediterrania.

FORNEROD, Anne (2019): "Jours fériés et fêtes religieuses", Revue du droit des religions, no 8, pp. 75-187. DOI : https://doi.org/10.4000/rdr.439

FREGOSI, Franck (2018): "De quoi le gouvernement de l'islam en France est-il le nom ?", Confluences Méditerranée, 106(3), pp. 35-51. DOI : https://doi.org/10.3917/come.106.0035

GARCIA-ROMERAL, Gloria y GRIERA, Mar (2011): "La construcció de I'islam com a objecte de polítiques públiques a Catalunya", Societat Catalana, pp. 231-253. 
GRIERA, Mar (2012): "Public policies, interfaith associations and religious minorities: a new policy paradigm? Evidence from the case of Barcelona", Social Compass, 59 (4), pp. 570-587. DOI: https://doi.org/10.1177/0037768612460800

GRIERA, Mar y CLOT-GARRELL, Anna (2015): "Banal is not Trivial: Visibility, Recognition, and Inequalities between Religious Groups in Prison", Journal of Contemporary Religion, 30 (1), pp. 2337. DOI: https://doi.org/10.1080/13537903.2015.986974

GRIERA, Mar y BURCHARDT, Marian (2016): “Religión y espacio público: el conflicto en torno a la regulación del velo integral islámico", Papeles del CEIC. International Journal on Collective Identity Research, no 2, pp. 1-28. DOI: http://dx.doi.org/10.1387/pceic.16190

GÖLE, Nilufer (2015): Musulmans au quotidien. Une enquête européenne sur les controverses autour de l'islam. Paris, La Découverte.

HAJJAT, Abdellalli y MOHAMMED, Marwan (2013): Islamophobie: comment les élits françaises fabriquent le "problème musulman ». Paris, La Découverte.

JELDFORT, Nadia. (2011): "Lived Islam: religious identity with 'nonorganized' Muslim minorities", Ethnic and Racial Studies, 34:7, pp. 1134-1151. DOI: https://doi.org/10.1080/01419870.2010.528441

KEPEL, Gilles. (2012) Quatre-vingt-treize. Paris, Ed. Gallimard.

LIOGIER, Raphael (2006): Une laïcité légitime. La France et ses religions d'État, Paris, MédicisEntrelacs.

LÓPEZ-BARGADOS, Alberto (2018) « Barcelona, ¿ciudad islamófoba? Variaciones sobre las políticas seculares y el "problema musulmán" ", Revista de Estudios Internacionales Mediterráneos, no 24, pp. 89-108. DOI: https://doi.org/10.15366/reim2018.24.006

MARTÍNEZ-ARIÑO, Julia (2018): “Conceptualising the role of cities in the governance of religious diversity in Europe", Current Sociology, 66 (5), pp. 810-827. DOI: https://doi.org/10.1177/0011392117745942

MARTINEZ-ARIÑO, Julia y GRIERA, Mar (2020): “Adapter la religion : négocier les limites de la religion minoritaire dans les espaces urbains", Social Compass, 67 (2), pp. 221-237. DOI: https://doi.org/10.1177/0037768620917085

MARTINEZ ARIÑO, Julia, GRIERA, Mar, GARCIA-ROMERAL, Gloria, FORTEZA, Maria (2011): "Inmigración, diversidad religiosa y centros de culto en la ciudad de Barcelona". Migraciones n 30 , pp. 101-103.

MORERAS, Jordi (2009): Una mesquita al barri. Conflicte, espai públic i inserció urbana dels oratoris musulmans a Catalunya, Fundació Jaume Bofill, disponible en: https://www.fbofill.cat/sites/default/files/1607.pdf [consulta: 24 de julio de 2020].

MORERAS, Jordi (dir.) (2017): Diàspores i rituals. El cicle festiu dels musulmans de Catalunya, Generalitat de Catalunya, Departament de Cultura.

PINAULT, David (1992): The Shiites: Ritual and popular piety in a Muslim community, New York, St. Martin's Press. 
PLANET, Ana (2018): Observing Islam in Spain: Contemporary Politics and Social Dynamics, Leiden, Brill.

ROY, Oliver (2013): Holy ignorance: When religion and culture part ways, Oxford, Oxford University Press.

SAINT-BLANCAT, Chantal (2019) : “Introduction. L'inscription religieuse dans l'espace urbain. État de l'art”, Social Compass, 66 (1), pp. 3-23. DOI: https://doi.org/10.1177/0037768618822275

SALGUERO MONTAÑO, Óscar (2018), “Baitul Mukarram: el islam en el espacio público del barrio de Lavapiés", Revista de Estudios Internacionales Mediterráneos, № 25, pp. 118-138. DOI: https://doi.org/10.15366/reim2018.25.007

SALGUERO MONTAÑO, Óscar y HEJAZI, Hután (2020): "El islam en el espacio público madrileño", Disparidades. Revista de Antropología, 75 (1). DOI: https://doi.org/10.3989/dra.2020.011

VÁSQUEZ, Manuel A., y KNOTT, Kim (2014): "Three dimensions of religious place making in diaspora", Global Networks, 14(3), pp. 326-347. DOI: https://doi.org/10.1111/glob.12062

VIVIER-MURESAN, Anne-Sophie (2020): "Rites d'Achoura et affirmations communautaires", Archives de sciences sociales des religions, 189(2), pp. 55-72. DOI: https://doi.org/10.4000/assr.49972

VOAS, David y FLEISCHMANN, Fenella (2012): "Islam moves west: Religious change in the first and second generations", Annual review of sociology, 38, pp. 525-54. DOI: https://doi.org/10.1146/annurev-soc-071811-145455 